\title{
The Effect of Nogood Recording in DPLL-CBJ SAT Algorithms
}

\author{
Inês Lynce and João Marques-Silva \\ Technical University of Lisbon, \\ IST/INESC/CEL, Lisbon, Portugal \\ \{ines, jpms\}@sat.inesc.pt
}

\begin{abstract}
Propositional Satisfiability (SAT) solvers have been the subject of remarkable improvements in the last few years. Currently, the most successful SAT solvers share a number of similarities, being based on backtrack search, applying unit propagation, and incorporating a number of additional search pruning techniques. Most, if not all, of the search reduction techniques used by state-of-the-art SAT solvers have been imported from the Constraint Satisfaction Problem (CSP) domain and, most significantly, include forms of backjumping and of nogood recording. This paper proposes to investigate the actual usefulness of these CSP techniques in SAT solvers, with the objective of evaluating the actual role played by each individual technique.
\end{abstract}

\section{Introduction}

The areas of Constraint Satisfaction Problem (CSP) and Propositional Satisfiability (SAT) have been the subject of intensive research in recent years, with significant theoretical and practical contributions. In the area of SAT, several highly optimized solvers have been developed 311/16/17/26]. These state-ofthe-art SAT solvers can now very easily solve very large, very hard real-world problem instances, which more traditional SAT solvers are totally incapable of. All of these highly effective SAT solvers are based on improvements made to the original Davis-Putnam-Logemann-Loveland (DPLL) backtrack search SAT algorithm [6]. Such improvements range from new search strategies, to new search pruning and reasoning techniques, and to new fast implementations.

Moreover, the relationship between SAT and CSP has become apparent due to an increasing number of mappings between SAT and CSP that have recently been proposed [10/24]. These different encodings have shed new insights on solving hard instances of CSP. Moreover, such results motivate a better understanding of the actual usefulness of the CSP techniques that have been utilized in successful SAT solvers.

Regarding different algorithmic solutions for SAT, and despite the potential theoretical and practical interest of all of them, we believe backtrack search to be the most robust approach for solving hard, structured, real-world instances of SAT. This belief has been amply supported by extensive experimental evidence obtained in recent years [31116 17/26]. Moreover, the most effective algorithms 
are complete, and so able to prove what local search is not capable of, i.e. unsatisfiability. Indeed, this is often the objective in a large number of significant SAT-related real-world applications.

Most if not all backtrack search SAT algorithms also incorporate propagation techniques for consistency checking, by applying Boolean constraint propagation (BCP) 25]. (Observe that backtrack search with BCP, i.e. DPLL, is conceptually similar to the maintaining arc consistency algorithm (MAC) [21], and equivalent for suitable mappings 11024.) Another strategy for reducing the number of searched nodes consists of performing back jumps in the search tree, skipping portions of the search space that can be shown not to contain a solution. In this context, and whenever a consistency check fails, conflict-directed backjumping (CBJ) [19] enables the search process to safely jump directly to the cause of the conflict.

In addition, state-of-the-art SAT solvers 311161726 effectively use learning techniques. In these solvers, whenever a conflict (dead-end) is reached, a new clause (nogood) is recorded to prevent the occurrence of the same conflict again during the subsequent search. Moreover, and from the first SAT solvers that incorporated non-chronological backtracking (NCB) 3 16], learning has always been a key component of the search algorithm, where recorded nogoods are used to determine the search point to backtrack to.

Hence, it is certainly relevant to conduct an unbiased evaluation of the isolated usefulness of DPLL-CBJ and of learning on a successful SAT solver. This work will allow us to find out whether a DPLL-CBJ SAT algorithm is enough per $s e$, or the algorithm should definitely include nogood learning techniques. Therefore, the objectives of this paper are two-fold. First, to describe the organization of a DPLL-CBJ SAT algorithm. Second, to evaluate the effect of learning in this algorithm. For this purpose, we developed a general framework that implements a DPLL-CBJ SAT algorithm with and without nogood recording. Moreover, we evaluate the performance on a representative set of instances, obtained from different real-world problems. This evaluation allows us to confirm and extend the preliminary experimental results presented in [2].

The remainder of the paper is organized as follows. Next, we introduce definitions used throughout the other sections. Moreover, we provide a historical perspective regarding the evolution of both CSP and SAT. Afterwards, we briefly describe chronological backtrack (CB) algorithms for SAT. In Section 4 we overview non-chronological backtracking (NCB) SAT algorithms, and further relate them with DPLL-CBJ. Experimental results are given in Section 5, and finally Section 6 concludes the paper.

\section{Background}

This section introduces the notational framework used throughout the paper, for CSP and SAT. Moreover, we provide a historical perspective for both areas. 


\section{$2.1 \quad$ CSP}

A CSP consists of a set of variables $V$ and a set of constraints $C$. Each variable $v \in V$ has a domain of values $M_{v}$ of size $m_{v}$. Each $a$-ary constraint $c \in C$ restricts a tuple of variables $\left\langle v_{1}, \ldots, v_{a}\right\rangle$ to an allowed combination of simultaneous values for the variables in the tuple. In a binary CSP, each constraint is a relation between two variables. Any binary CSP can be associated with a constraint graph, where the nodes represent variables and each edge links a pair of nodes if and only if there is a constraint on the corresponding variables. A CSP consists of deciding whether there exists a (consistent) assignment to the variables such that all the constraints are satisfied, i.e. no $c \in C$ is violated.

In tree search algorithms for CSP the variables are incrementally instantiated with values from their respective domains. In chronological backtrack (CB), when a value is assigned to a variable, consistency checking is performed backwards against the already instantiated variables. If a conflict (dead-end) is reached, the algorithm backtracks to the most recent (not wiped-out1) assigned variable, changes its assignment and continues from there.

Trying to improve the performance of backtrack search entails deciding how far to backtrack and also recording the reasons for the dead-end in the form of new constraints (nogoods). The idea of going back several levels in a dead-end situation, rather than going back to the chronologically most recent decision, was exploited independently in [9], where the term backjumping (BJ) was introduced, and in [23] as a part of the dependency-directed backtracking algorithm. Another example is Dechter's graph-based backjumping (GBJ) [8] that proposes to jump back to the source of the failure by using knowledge extracted from the constraint graph. In addition, conflict-directed backjumping (CBJ) [19] consists of keeping a set of past variables that failed consistency checks with each variable, based on dependencies from the constraints.

Arc-consistency (AC) [15] is a polynomial propagation algorithm commonly used in CSP. A state is arc-consistent if every variable has a value in its domain that is consistent with each of the constraints on that variable. Arc consistency can be achieved by successive deletion of values that are inconsistent with some constraint. As values are deleted, other values may become inconsistent because they relied on the deleted values. Arc consistency therefore exhibits a form of constraint propagation, as choices are gradually narrowed down. Furthermore, maintaining arc consistency (MAC) 21] is a solution procedure which incorporates and further maintains arc consistency during backtrack search. In addition, MAC can be improved by adding conflict-directed backjumping (CBJ), thus obtaining the algorithm MAC-CBJ [20].

\subsection{SAT}

In a SAT problem, propositional variables are denoted $x_{1}, \ldots, x_{n}$, and can be assigned truth values 0 ( or $F$ ) or 1 (or $T$ ). The truth value assigned to a variable $x$

\footnotetext{
${ }^{1}$ A variable domain is wiped-out when all values have been tried for that variable without success.
} 
is denoted by $\nu(x)$. (When clear from context we use $x=\nu_{x}$, where $\nu_{x} \in\{0,1\}$ ). A literal $l$ is either a variable $x_{i}$ or its negation $\neg x_{i}$. A clause $\omega$ is a disjunction of literals and a CNF formula $\varphi$ is a conjunction of clauses. A clause is said to be satisfied if at least one of its literals assumes value 1, unsatisfied if all of its literals assume value 0 , unit if all but one literal assume value 0 , and unresolved otherwise. Literals with no assigned truth value are said to be free literals. A formula is said to be satisfied if all its clauses are satisfied, and is unsatisfied if at least one clause is unsatisfied. A truth assignment $A$ for a formula is a set of assigned variables and their corresponding truth values. The SAT problem consists of deciding whether there exists a truth assignment to the variables such that the formula becomes satisfied.

Over the years a large number of algorithms has been proposed for SAT, from the original Davis-Putnam procedure (DP) [7], to recent backtrack search algorithms [312 16]17 26], to local search algorithms [22], among many others. Among the different algorithms, we believe backtrack search to be the most robust approach for solving hard, structured, real-world instances of SAT. Observe that only complete algorithms can establish unsatisfiability if given enough CPU time, which is often a requirement when considering real-world instances.

The vast majority of backtrack search SAT algorithms build upon the original backtrack search algorithm of Davis, Logemann and Loveland (DPLL) [6]. Moreover, non-chronological backtracking strategies (NCB) attempt to identify the variable assignments causing a conflict and backtrack directly to a point so that at least one of those variable assignments is modified. GRASP [16] and relsat [3] are examples of SAT solvers that successfully implement non-chronological backtracking.

Recent state-of-the-art SAT solvers are also characterized by using very efficient data structures, intended to reduce the CPU time required per each node in the search tree. Examples of efficient lazy data structures include the head/tail lists used in SATO [26] and the watched literals used in Chaff [17.

\section{Chronological Backtrack SAT Algorithms}

A backtrack search SAT algorithm is implemented by a search process that implicitly enumerates the space of $2^{n}$ possible binary assignments to the $n$ problem variables. Each different truth assignment defines a search path within the search space. A decision level is associated with each variable selection and assignment. The first variable selection corresponds to decision level 1, and the decision level is incremented by 1 for each new variable decision assignment? When relevant to the context, we use an assignment notation to indicate the decision level at which the variable assignment occurred. Thus, $x=\nu_{x} @ d$ reads as " $x$ is assigned $\nu_{x}$ at decision level $d "$ ". In addition, and for each decision level, the unit clause rule [7] is applied. If a clause is unit, then the sole free literal must be assigned value 1 for the formula to be satisfied. In this case, the value of the literal and of

\footnotetext{
${ }^{2}$ Observe that all the assignments made before the first decision assignment correspond to decision level 0 .
} 
the associated variable are said to be implied. The iterated application of the unit clause rule is often referred to as Boolean Constraint Propagation (BCP) [25].

In chronological backtracking (CB), the search algorithm keeps track of which decision assignments have been toggled. Given an unsatisfied clause (i.e. a conflict or a dead end) at decision level $d$, the algorithm checks whether at the current decision level the corresponding decision variable $x$ has already been toggled. If not, the algorithm erases the variable assignments which are implied by the assignment on $x$, including the assignment on $x$, assigns the opposite value to $x$, and marks decision variable $x$ as toggled. In contrast, if the value of $x$ has already been toggled, the search backtracks to decision level $d-1$.

\section{Non-chronological Backtrack SAT Algorithms}

All of the most effective recent state-of-the-art SAT solvers [3 1116 16 26] utilize different forms of non-chronological backtracking (NCB). Non-chronological backtracking backs up the search tree to one of the identified causes of failure, skipping over irrelevant variable assignments.

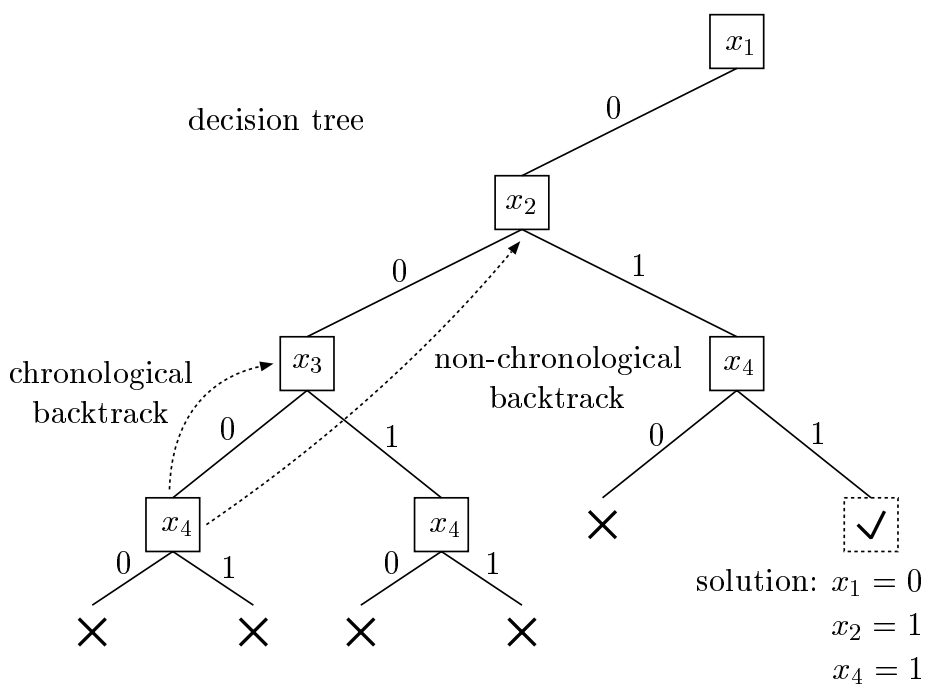

Fig. 1. Non-Chronological Backtracking

For example, let us consider Figure 1, that illustrates non-chronological backtracking for a given CNF formula. Once both $x_{1}$ and $x_{2}$ are assigned value 0 , there are no possible assignments for the remaining variables $x_{3}$ and $x_{4}$ that can satisfy the formula. In this example, chronological backtracking wastes a potentially significant amount of time exploring a region of the search space without 
solutions, only to discover, after potentially much effort, that the region does not contain any satisfying assignments.

The forms of non-chronological backtracking used in state-of-the-art SAT solvers are related to dependency-directed backtracking [23], since they are always associated with learning from conflicts. The incorporation of learning consists of the following: for each identified conflict, its causes are identified, and a new clause (called nogood) is created to explain and subsequently prevent the identified conflicting conditions.

In the next section we address conflict-directed backjumping (CBJ) [19, another form of non-chronological backtracking that does not incorporate learning. Afterwards, we describe the use of conflict-directed backjumping jointly with learning.

\subsection{Conflict-Directed Backjumping}

Conflict-directed backjumping (CBJ) [19] is the most accurate form of backjumping, and can be considered a combination of Gaschnig's backjumping (BJ) [9] and Dechter's graph-based backjumping (GBJ) [8].

BJ aims performing higher jumps in the search tree, rather than backtracking to the most recent yet untoggled decision variable. For each value of a variable $v_{j}$, Gaschnig's algorithm obtains the lowest level for which the considered assignment is inconsistent. In addition, BJ uses a marking technique that maintains, for each variable $v_{j}$, a reference to a variable $v_{i}$ with the deepest level of the different levels with which any value of $v_{j}$ was found to be inconsistent. Hence, a backjump from $v_{j}$ is to $v_{i}$. Moreover, if the domain of $v_{i}$ is wiped-out, then the search must chronologically backtrack to $v_{i-1} \cdot \mathrm{q}$

As an improvement, Dechter's GBJ extracts knowledge about dependencies from the constraint graph. CBJ builds upon this idea and, based on dependencies from the constraints, records the set of past variables that failed consistency checks with each variable $v$. This set (called conflict set in 8]) allows the algorithm to perform multiple jumps.

\subsection{Learning and Conflict-Directed Backjumping}

Learning can be combined with CBJ when each identified conflict is analyzed, its causes are identified, and a nogood is recorded to explain and prevent the identified conflicting conditions from occurring again during the subsequent search. Moreover, the newly recorded nogood is then used to compute the backtrack point as the most recent decision assignment from all the decision assignments represented in the recorded nogood.

For implementing learning techniques common to some of the most competitive backtrack search SAT algorithms, it is necessary to properly explain the truth assignments to the propositional variables that are implied by the clauses of the CNF formula. For example, let $x=v_{x}$ be a truth assignment implied by applying the unit clause rule to a unit clause $\omega$. Then the explanation for this 


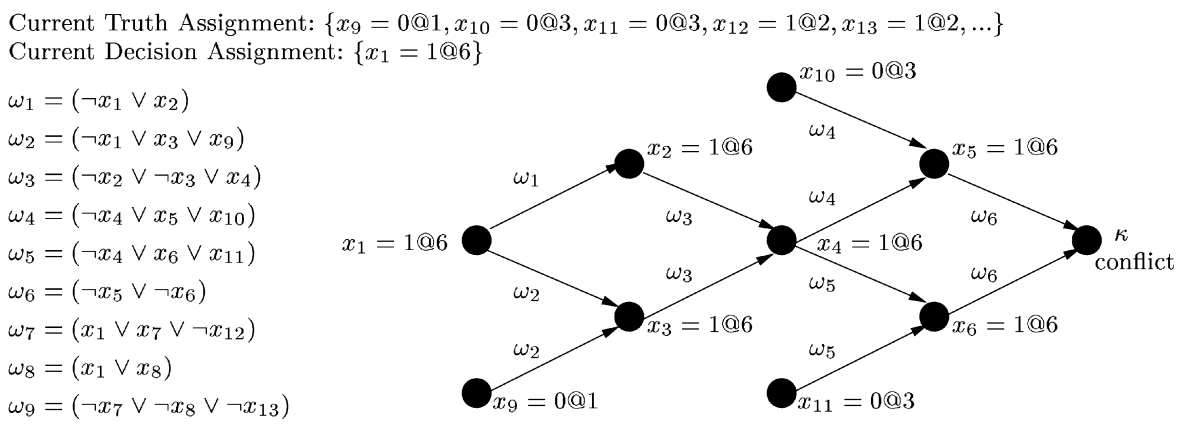

Fig. 2. Example of conflict diagnosis with nogood recording

assignment is the set of assignments associated with the remaining literals of $\omega$, which are assigned value 0 . For example, let $\omega=\left(x_{1} \vee \neg x_{2} \vee x_{3}\right)$ be a clause of a $\mathrm{CNF}$ formula $\varphi$, and assume the truth assignments $\left\{x_{1}=0, x_{3}=0\right\}$. For clause $\omega$ to be satisfied we must necessarily have $x_{2}=0$. Hence, we say that the antecedent assignment of $x_{2}$, denoted as $A\left(x_{2}\right)$, is defined as $A\left(x_{2}\right)=\left\{x_{1}=0, x_{3}=0\right\}$.

In addition, in order to explain other NCB-related concepts, we shall often analyze the directed acyclic implication graph created by the sequences of implied assignments generated by BCP. An implication graph $I$ is defined as follows:

1. Each vertex in $I$ corresponds to a variable assignment at a given decision level $x=\nu(x) @ d$.

2. The predecessors of vertex $x=\nu(x)$ in $I$ are the antecedent assignments $A(x)$ corresponding to the unit clause $\omega$ that caused the value of $x$ to be implied. The directed edges from the vertices in $A(x)$ to vertex $x=\nu(x)$ are all labeled with $\omega$. Vertices that have no predecessors correspond to decision assignments.

3. Special conflict vertices are added to $I$ to indicate the occurrence of conflicts. The predecessors of a conflict vertex $\kappa$ correspond to variable assignments that force a clause $\omega$ to become unsatisfied and are viewed as the antecedent assignment $A(\kappa)$. The directed edges from the vertices in $A(\kappa)$ to $\kappa$ are all labeled with $\omega$.

Next, we illustrate nogood recording with the example of Figure 2 A subset of the CNF formula is shown, and we assume that the current decision level is 6 , corresponding to the decision assignment $x_{1}=1$. This assignment yields a conflict $\kappa$ involving clause $\omega_{6}$. By inspection of the implication graph, we can readily conclude that a sufficient condition for this conflict to be identified is,

$$
\left(x_{10}=0\right) \wedge\left(x_{11}=0\right) \wedge\left(x_{9}=0\right) \wedge\left(x_{1}=1\right)
$$

By creating clause $\omega_{10}=\left(x_{10} \vee x_{11} \vee x_{9} \vee \neg x_{1}\right)$ we prevent the same set of assignments from occurring again during the subsequent search. 


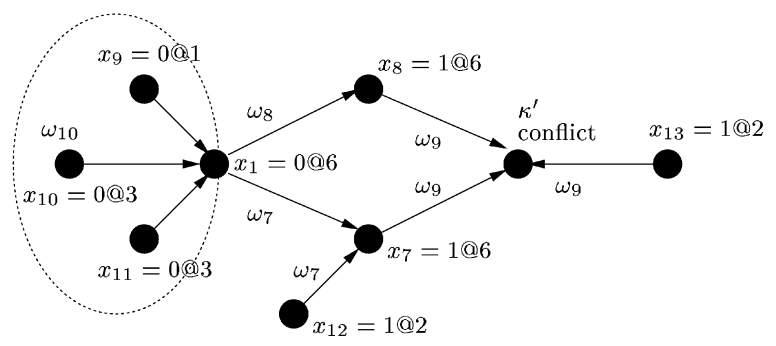

(a) Conflicting implication sequence

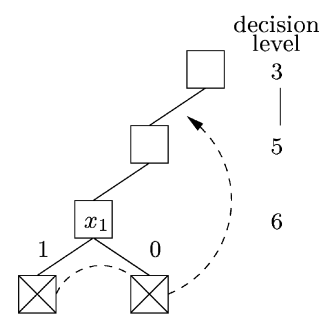

(b) Decision tree

Fig. 3. Computing the backtrack decision level

In order to illustrate non-chronological backtracking based on nogood recording, let us now consider the example of Figure 3, which continues the example in Figure 2, after recording clause $\omega_{10}=\left(x_{10} \vee x_{11} \vee x_{9} \vee \neg x_{1}\right)$. At this stage BCP implies the assignment $x_{1}=0$ because clause $\omega_{10}$ becomes unit at decision level 6. By inspection of the CNF formula (see Figure 2), we can conclude that clauses $\omega_{7}$ and $\omega_{8}$ imply the assignments shown, and so we obtain a conflict $\kappa^{\prime}$ involving clause $\omega_{9}$. By creating clause $\omega_{11}=\left(\neg x_{13} \vee \neg x_{12} \vee x_{11} \vee x_{10} \vee x_{9}\right)$ we prevent the same conflicting conditions from occurring again. It is straightforward to conclude that even though the current decision level is 6, all assignments directly involved in the conflict are associated with variables assigned at decision levels less than 6 , the highest of which being 3 . Hence we can backtrack immediately to decision level 3.

\subsection{Nogood Deletion Policy}

Unrestricted nogood recording can in some cases be impractical. Recorded nogoods consume memory and repeated recording of nogoods can eventually lead to the exhaustion of the available memory. Observe that the number of recorded nogoods grows with the number of conflicts; in the worst case, such growth can be exponential in the number of variables. Furthermore, large recorded nogoods are known for not being particularly useful for search pruning purposes [16]. Adding larger nogoods leads to additional overhead for conducting the search process and, hence, it eventually costs more than what it saves in terms of backtracks.

As a result, there are three main solutions for guaranteeing the worst case growth of the recorded nogoods to be polynomial in the number of variables [14]:

1. We may consider $n$-order learning, that records only nogoods with $n$ or fewer literals [8].

2. Nogoods can be temporarily recorded while they either imply variable assignments or are unit clauses, being discarded as soon as the number of unassigned literals is greater than an integer $m$. This technique is named $m$-size relevance-based learning [3]. 
3. Nogoods with a size less than a threshold $k$ are kept during the subsequent search, whereas larger nogoods are discarded as soon as the number of unassigned literals is greater than one. We refer to this technique as $k$-bounded learning [16].

Observe that we can combine $k$-bounded learning with $m$-size relevance-based learning. The search algorithm is organized so that all recorded nogoods of size no greater than $k$ are kept and larger nogoods are deleted only after $m$ literals have become unassigned.

More recently, a heuristic nogood deletion policy has been introduced [1]. Basically, the decision whether a nogood should be deleted is based not only on the number of literals but also on its activity in contributing to conflict making and on the number of decisions taken since its creation.

\section{$5 \quad$ Experimental Results}

In this section we present the obtained experimental results. We start by describing the experimental setup that has been used for the different results. Then we analyze the results for the DPLL-CB SAT algorithm, the DPLL-CBJ SAT algorithm and the DPLL-CBJ SAT algorithm with nogood recording.

\subsection{Experimental Setup}

In order to experimentally evaluate the different algorithms, in a controlled experiment that ensures that only specific differences are evaluated, a dedicated SAT solving framework is needed. Consequently, we developed the JQUEST SAT framework, a Java implementation that can be used to conduct unbiased experimental evaluations of SAT algorithms and techniques.

This comparison was performed using the JQUEST SAT solver on instances selected from several classes of instances (see Table 1) 3 . In all cases, the problem instances chosen can be solved with several thousands of decisions by the most effective solvers, usually taking a few tens of seconds, thus being significantly hard. For this reason, different algorithms can result in significant variations on the time required for solving a given instance. In addition, we should also observe that the problem instances selected are intended to be representative, since each resembles, in terms of hardness for SAT solvers, the typical instance in each class of problem instances.

For the results shown a P-IV@1.7 GHz Linux machine with 1 GByte of physical memory was used. The Java Virtual Machine used was SUN's HotSpot JVM for JDK1.4. The CPU time was limited to 1500 seconds.

\footnotetext{
${ }^{3}$ All the instances are available from http://www.lri.fr/ $\sim$ simon/satex/satex.php3 (Sat-Ex web site), with the exception of the superscalar processor verification instances.
} 
Table 1. Example Instances

\begin{tabular}{|c|c|c|c|c|}
\hline Application Domain & Selected Instance & \# Variable & \#Clauses & Satisfiable? \\
\hline \multirow{2}{*}{$\begin{array}{l}\text { Circuit Testing } \\
(\text { Dimacs })\end{array}$} & bf0432-079 & 1044 & 3685 & $\mathrm{~N}$ \\
\hline & ssa2670-141 & 4843 & 2315 & $\mathrm{~N}$ \\
\hline \multirow{2}{*}{$\begin{array}{l}\text { Inductive } \\
\text { Inference(Dimacs) }\end{array}$} & ii16b2 & 1076 & 16121 & $\overline{\mathrm{Y}}$ \\
\hline & ii16e1 & 1245 & 14766 & $\mathrm{Y}$ \\
\hline \multirow{2}{*}{$\begin{array}{l}\text { Parity } \\
\text { Learning(Dimacs) }\end{array}$} & par16-1-c & 317 & 1264 & $\mathrm{Y}$ \\
\hline & par16-4 & 1015 & 3324 & $\mathrm{Y}$ \\
\hline \multirow{2}{*}{ Graph Colouring } & flat200-39 & 600 & 2237 & $\mathrm{Y}$ \\
\hline & sw100-49 & 500 & 3100 & $\mathrm{Y}$ \\
\hline \multirow{2}{*}{ Quasigroup } & qg3-08 & 512 & 10469 & $\overline{\mathrm{Y}}$ \\
\hline & qg5-09 & 729 & 28540 & $\mathrm{~N}$ \\
\hline \multirow{2}{*}{ Blocks World } & 2bitadd_12 & 708 & 1702 & $\bar{Y}$ \\
\hline & 4blocksb & 410 & 24758 & $\mathrm{Y}$ \\
\hline \multirow{2}{*}{ Planning-Sat } & logistics.a & 828 & 6718 & $\overline{\mathrm{Y}}$ \\
\hline & bw_large.c & 3016 & 50457 & $\mathrm{Y}$ \\
\hline \multirow{2}{*}{ Planning-Unsat } & logistics.c & 1027 & 9507 & $\mathrm{~N}$ \\
\hline & bw_large.b & 920 & 11491 & $\mathrm{~N}$ \\
\hline \multirow{3}{*}{$\begin{array}{l}\text { Bounded Model } \\
\text { Checking }\end{array}$} & barrel5 & 1407 & 5383 & $\mathrm{~N}$ \\
\hline & queueinvar16 & 1168 & 6496 & $\mathrm{~N}$ \\
\hline & longmult6 & 2848 & 8853 & $\mathrm{~N}$ \\
\hline \multirow{4}{*}{$\begin{array}{l}\text { Superscalar } \\
\text { Processor } \\
\text { Verification }\end{array}$} & dlx2_aa & 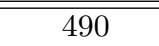 & 2804 & $\overline{\mathrm{N}}$ \\
\hline & dlx2_cc_a_bug17 & 4847 & 39184 & $\mathrm{Y}$ \\
\hline & 2dlx_cc_mc_ex_bp_f2_bug006 & 4824 & 48215 & $\mathrm{Y}$ \\
\hline & 2dlx_cc_mc_ex_bp_f2_bug010 & 5754 & 60689 & $\mathrm{Y}$ \\
\hline \multirow{2}{*}{$\begin{array}{l}\text { Data Encryption } \\
\text { Standard }\end{array}$} & cnf-r3-b2-k1.1 & 5679 & 17857 & $\mathrm{Y}$ \\
\hline & cnf-r3-b4-k1.2 & 2855 & 35963 & $\mathrm{Y}$ \\
\hline
\end{tabular}

\subsection{CBJ and Nogood Recording}

The first table of results (Table 2) shows the CPU time required to solve each problem instance 4 . For the algorithms considered: CB denotes the chronological backtracking search SAT algorithm (corresponding to DPLL), CBJ denotes the DPLL-CBJ SAT algorithm and $\mathbf{C B J + n g}$ denotes the CBJ SAT algorithm with nogood recording. Moreover, a variety of nogood deletion policies were considered, depending on the value of $k$, where $k$ defines the $k$-bounded learning procedure used (see Section 4.3). For instance, +ng10 means that recorded nogoods with size greater than 10 are deleted as soon as they become unresolved (i.e. not satisfied with more than one unassigned literal), whereas +ngAll means that all the recorded nogoods are kept.

Table 2 reveals interesting trends, and several conclusions can be drawn:

- Clearly, CB and CBJ have in general similar behavior (except for bf0432-079 and data encryption standard instances, that only CBJ is able to solve).

${ }^{4}$ Instances that were not solved in the allowed CPU time are marked with —. 
Table 2. CPU Time (in seconds)

\begin{tabular}{|c|c|c|c|c|c|c|c|c|c|}
\hline \multirow[t]{2}{*}{ Instance } & \multirow{2}{*}{ CB } & \multirow{2}{*}{ CBJ } & \multicolumn{7}{|c|}{ CBJ+ng } \\
\hline & & & $+\mathrm{ng} 0$ & + ng5 & +ng10 & +ng20 & + ng50 & +ng100 & + ngAll \\
\hline 104 & & 1.74 & 5.18 & 2.78 & 2.97 & 2.70 & .53 & 1.44 & 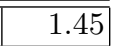 \\
\hline ssa2t & 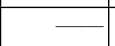 & - & 1.21 & .87 & 0.81 & 0.52 & 0.55 & 0.54 & 0.5 \\
\hline$\overline{\text { ii16b }}$ & 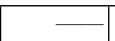 & 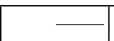 & 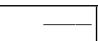 & - & 857.61 & 302.63 & 158.63 & 141.41 & $\overline{141.0}$ \\
\hline ii16e & & 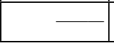 & 20.58 & 26.75 & 20.40 & 12.65 & 12.89 & 15.96 & 11.8 \\
\hline par16-1-c & 5.92 & 7.06 & 19.91 & 14.75 & 16.07 & 16.78 & 18.19 & 18.08 & $\overline{8.1}$ \\
\hline par16 & 4.88 & 0.59 & 11.51 & 8.49 & 8.77 & 9.34 & 7.16 & 7.20 & 7.14 \\
\hline flat2 & .44 & 8.75 & 97.35 & 255.04 & 85.19 & 114.05 & 67.55 & 67.00 & 67.19 \\
\hline sw10 & $\square$ & 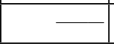 & 1.94 & 13.48 & 1.26 & 2.18 & 0.73 & 0.74 & 0.7 \\
\hline qg3- & 29 & 2.65 & 0.86 & 0.88 & 0.91 & 1.00 & 1.07 & 1.30 & 1.3 \\
\hline q95-09 & 3.28 & 8.61 & 1.35 & 1.29 & 1.06 & 1.17 & 1.16 & 1.21 & 1.15 \\
\hline 2 2bitadd & - & - & 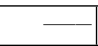 & $\square$ & $\square$ & - & 87.68 & 50.74 & 50.9 \\
\hline $4 \mathrm{bloc}$ & - & 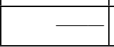 & 31.23 & 30.25 & 39.62 & 29.66 & 16.34 & 20.33 & 31.7 \\
\hline logis & 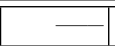 & - & 2.98 & 1.87 & 1.62 & 1.65 & 1.61 & 1.63 & 1.6 \\
\hline bw_la & - & & 76.03 & 55.13 & 36.41 & 38.37 & 43.71 & 38.03 & 38.0 \\
\hline logis & - & - & 26.88 & 7.24 & 4.60 & 15.40 & 10.22 & 10.23 & $\overline{10.2}$ \\
\hline bw_large.b & 8.27 & 4.78 & 1.60 & 0.59 & 0.61 & 0.64 & 0.61 & 0.62 & 0.6 \\
\hline barrel5 & 9.49 & 32.37 & 171.94 & 279.31 & 36.35 & 19.80 & 23.43 & 24.00 & 21.99 \\
\hline queueinvar & $\square$ & 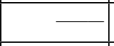 & 23.36 & 21.39 & 15.74 & 15.48 & 8.05 & 8.08 & 8.1 \\
\hline longmult6 & 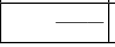 & - & 27.66 & 23.63 & 26.20 & 29.16 & 32.32 & 31.74 & 32.02 \\
\hline dlx2_aa & 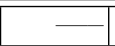 & 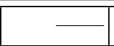 & 54.74 & 36.21 & 37.96 & 9.80 & 6.43 & 6.62 & 6.60 \\
\hline dlx2_cc_a_bug17 & 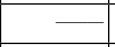 & & 430.31 & $\square$ & Z & 500.25 & 220.06 & 6.48 & 6.54 \\
\hline 2 dlx_..._bug006 & 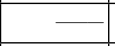 & 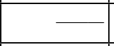 & 17.29 & 14.32 & 3.87 & 2.27 & 2.27 & 2.23 & 2.22 \\
\hline $2 \mathrm{dlx}_{\text {_..._b_bug010 }}$ & 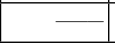 & & 3.32 & 4.13 & 3.83 & 5.31 & 4.55 & 2.03 & 1.93 \\
\hline cnf-r3-b2-k1.1 & 2.90 & 9.37 & 3.05 & 3.13 & 2.39 & 2.58 & 2.40 & 2.16 & 3.90 \\
\hline cnf-r3-b4-k1.2 & 405.98 & 12.62 & 5.42 & 5.39 & 5.48 & 5.12 & 4.89 & 4.45 & 3.91 \\
\hline
\end{tabular}

- The CBJ+ng algorithms are in general clearly more efficient than the other algorithms. Indeed, for almost all the instances CBJ+ng achieves remarkable improvements, when compared with CB or with CBJ. Instances flat200-39 and barrel5 are the only exceptions. (For instance barrel5, this is only true for CBJ+ng with small values of $k$.)

- Some of the instances that are not solved by CBJ in the allowed CPU time (e.g ii16b2 and dlx2_cc_a_bug17), also need a significant amount of time to be solved by $k$-bounded learning with a small value of $k$.

- For instance flat200-39, recorded nogoods result in an additional search effort to find a solution.

- From a practical perspective, unrestricted nogood recording is not necessarily a bad approach. 
Table 3. Searched nodes

\begin{tabular}{|c|c|c|c|c|c|c|c|c|c|}
\hline \multirow[t]{2}{*}{ Instance } & \multirow{2}{*}{ CB } & \multirow{2}{*}{ CBJ } & \multicolumn{7}{|c|}{ CBJ+ng } \\
\hline & & & $+n g 0$ & +ng5 & +ng10 & +ng20 & +ng50 & +ng100 & $+n g A$ \\
\hline$\overline{\mathrm{bf043}}$ & 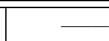 & \begin{tabular}{|c|}
98824 \\
\end{tabular} & 3939 & $\bar{~} 1950$ & 2010 & 1600 & 1168 & 1188 & $\overline{1188}$ \\
\hline$\overline{\text { ssa26 }}$ & 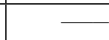 & & 2882 & 1988 & 1480 & 806 & 736 & 698 & 698 \\
\hline$\overline{\text { ii16b2 }}$ & 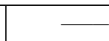 & - & 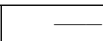 & 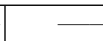 & 101451 & 32923 & \begin{tabular}{|l|l|}
3 & 12188 \\
\end{tabular} & 10573 & 10573 \\
\hline ii16e1 & 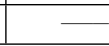 & $\square$ & 20872 & 24714 & 17271 & 13869 & 8117 & 8442 & 7869 \\
\hline par16-1-c & $\overline{2800}$ & 52800 & 11307 & $\overline{7249}$ & 7524 & 5255 & 5364 & 5364 & $\overline{5364}$ \\
\hline par16-4 & 0673 & 10246 & 4157 & 2676 & 2745 & 2467 & 1919 & 1919 & 1919 \\
\hline flat200-39 & 6286 & 5427 & 139264 & 287983 & 51308 & 40888 & \begin{tabular}{|l|l|}
36428 \\
\end{tabular} & 25738 & 25738 \\
\hline sw100-49 & - & - & 4596 & 44247 & 2370 & 3748 & \begin{tabular}{|l|l|}
3 & 1450 \\
\end{tabular} & 1450 & 1450 \\
\hline qg3-08 & 703 & 703 & 220 & 220 & 242 & 214 & 222 & 282 & 282 \\
\hline qg5-09 & 1578 & 1547 & 373 & 329 & 318 & 337 & 337 & 337 & 337 \\
\hline 2bitadd_12 & - & 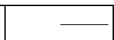 & - & $\longrightarrow$ & 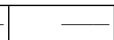 & 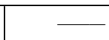 & 21244 & 11238 & 11238 \\
\hline 4blocksb & 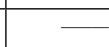 & 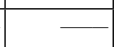 & 5559 & 5007 & 6205 & 5009 & 2618 & 2491 & 3363 \\
\hline logistics.a & - & - & 32872 & 16999 & 14899 & 15185 & 15185 & 15185 & 15185 \\
\hline bw_large.c & 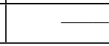 & - & 6137 & 5132 & 2763 & 2878 & \begin{tabular}{|l|l|}
3000 \\
\end{tabular} & 2783 & 2783 \\
\hline logistics.c & $\bar{~}$ & $\bar{~}$ & $\overline{\overline{55721}}$ & $\bar{~} 18839$ & 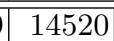 & 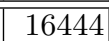 & 15441 & 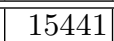 & $\bar{~} 15441$ \\
\hline bw_large.b & 1431 & 1112 & 293 & 128 & 195 & 195 & 195 & 195 & 195 \\
\hline barrel5 & 24727 & 24664 & 90115 & 141953 & 14684 & 8731 & 10396 & 12315 & 5985 \\
\hline queueinvar16 & - & - & 45053 & 41510 & 24842 & 19557 & 8460 & 8506 & 8083 \\
\hline longmult6 & - & - & 7407 & 5482 & 5666 & 5507 & 5019 & 4729 & 4725 \\
\hline dlx2_aa & - & & 319120 & 204995 & 208725 & 21036 & 10062 & 10035 & 10035 \\
\hline dlx2_cc_a_bug17 & - & - & 446626 & - & ב & 212816 & 85713 & 3383 & 3383 \\
\hline $2 \mathrm{dlx}_{\text {_...._bug006 }}$ & 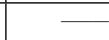 & - & 32297 & 25259 & 7775 & 3288 & \begin{tabular}{|l|l|}
3227 \\
\end{tabular} & 3123 & 3123 \\
\hline 2 dlx_..._bug010 & - & & 10086 & 19002 & 14358 & 13229 & 8533 & 3547 & 3522 \\
\hline cnf-r3-b2-k1.1 & 219037 & 6273 & 1168 & 1138 & 872 & 942 & 825 & 667 & 1221 \\
\hline cnf-r3-b4-k1.2 & 57843 & 2216 & 1011 & 1012 & 1010 & 943 & 910 & 776 & 729 \\
\hline
\end{tabular}

It is interesting to observe that the uselessness of CBJ-related algorithms w.r.t. CB-related algorithms has been experienced in the past [4]18. CBJ, when applied jointly with a domain filtering procedure (e.g. AC) and an accurate variable ordering heuristic, has been considered an expensive approach that almost always slows down the search, even when it saves a few constraint checks 5 .

Table 3 gives the results for the number of searched nodes, for each instance and for the different configurations. It is plain from the results that $\mathrm{CB}$ and CBJ in general need to search more nodes to find a solution than the other algorithms. This can be explained by the effect of the recorded nogoods. Besides explaining an identified conflict, nogoods are often re-used, either for yielding conflicts or for implying variable assignments, introducing significant pruning in

${ }^{5}$ However, different conclusions have been obtained for specific classes of instances [5]. 
the search tree. Moreover, other conclusions can be established from the results on the searched nodes:

- For the par instances, CB and CBJ have the same or an approximate number of search nodes for these instances. This is explained by the fact that there are none or just a few backjumps during the search.

- For instances logistics.a, bw_large.b and qg5-09 the search needs the same number of nodes for increasing values of $k$, since only small-size nogoods are recorded.

- Usually more recorded nogoods imply less searched nodes and less time needed to find a solution. (Even though the reduction in the number of nodes is more significant than the reduction in the amount of time, due to the overhead introduced by the management of nogoods.)

Overall, the effect of nogood recording is clear, and in general dramatic. The results clearly indicate that nogood recording is an essential component of current state-of-the-art SAT solvers. Nevertheless, the actual role played by the value of $k$ is not clear, and subject of additional research.

As a final remark, we evaluated whether a different variable ordering heuristic could have affected the result 6 . The intuition was that a more elaborate heuristic could have improved the results obtained for CB and CBJ. Nevertheless, the experimental results presented in [13] for CB and CBJ, that include a more sophisticated heuristic, are still far from being competitive with non-chronological backtracking with nogood recording.

\section{Conclusions and Future Directions}

In this paper we address the use of DPLL-CBJ in SAT algorithms. In addition, we evaluate the effect of nogood recording in DPLL-CBJ SAT algorithms, and further analyze the effect of different nogood deletion policies. Given the experimental results, obtained for representative instances from several classes of problem instances, we conclude that nogood recording is crucial for competitive SAT algorithms. In addition, the results strongly suggest that backjumping techniques are not enough per se for state-of-the-art SAT solvers.

Moreover, we believe that CSP algorithms may also improve their performance by applying both jumping and learning. Interestingly, backjumping techniques and learning have their roots in Truth Maintenance Systems 23] but have been extensively studied in CSP 8919; nevertheless, constraint programming technology appears not to exploit it.

Future research work will extend the results of this paper by considering alternative approaches with the goal of optimizing SAT solvers. It is well-known that

\footnotetext{
${ }^{6}$ For the above results, we have applied the variable selection heuristic VSIDS (Variable State Independent Decaying Sum) [17]. It selects the literal that appears most frequently over all clauses, which means that the metrics only have to be updated when a new recorded clause is created.
} 
state-of-the-art SAT solvers use nogood recording. On the other hand, DPLLCBJ does not incorporate learning, but does consider conflict sets. Hence, we can envision an algorithm that explores the advantages of CBJ to compensate the disadvantages of nogood recording. This algorithm can apply nogood recording, but use conflict sets to avoid recording large nogoods that must be eventually deleted.

Acknowledgments. We would like to thank Patrick Prosser for the insightful discussions we had on CBJ. This work is partially supported by the European research project IST-2001-34607 and by Fundação para a Ciência e Tecnologia under research projects PRAXIS/C/EEI/11249/98 and POSI/34504/CHS/2000.

\section{References}

1. K. Apt. Some remarks on boolean constraint propagation. In New Trends in Constraints, number 1865 in Lecture Notes in Artificial Intelligence, pages 91-107. Springer, 2000.

2. R. Bayardo Jr. and R. Schrag. Using CSP look-back techniques to solve exceptionally hard SAT instances. In Proceedings of the International Conference on Principles and Practice of Constraint Programming, pages 46-60, August 1996.

3. R. Bayardo Jr. and R. Schrag. Using CSP look-back techniques to solve real-world SAT instances. In Proceedings of the National Conference on Artificial Intelligence, pages 203-208, July 1997.

4. C. Bessière and J. C. Régin. MAC and combined heuristics: two reasons to forsake FC (and CBJ?) on hard problems. In Proceedings of the International Conference on Principles and Practice of Constraint Programming, pages 61-75, August 1996.

5. X. Chen and P. van Beek. Conflict-directed backjumping revisited. Journal of Artificial Intelligence Research, 14:53-81, 2001.

6. M. Davis, G. Logemann, and D. Loveland. A machine program for theoremproving. Communications of the Association for Computing Machinery, 5:394-397, July 1962.

7. M. Davis and H. Putnam. A computing procedure for quantification theory. Journal of the Association for Computing Machinery, 7:201-215, July 1960.

8. R. Dechter. Enhancement schemes for constraint processing: backjumping, learning, and cutset decomposition. Artificial Intelligence, 41(3):273-312, January 1990.

9. J. Gaschnig. Performance Measurement and Analysis of Certain Search Algorithms. PhD thesis, Carnegie-Mellon University, Pittsburgh, PA, May 1979.

10. I. Gent. Arc consistency in SAT. In Proceedings of the European Conference on Artificial Intelligence, pages 121-125, July 2002.

11. E. Goldberg and Y. Novikov. BerkMin: a fast and robust sat-solver. In Proceedings of the Design and Test in Europe Conference, pages 142-149, March 2002.

12. C. M. Li and Anbulagan. Look-ahead versus look-back for satisfiability problems. In Proceedings of the International Conference on Principles and Practice of Constraint Programming, pages 341-355, October 1997.

13. I. Lynce and J. P. Marques-Silva. The effect of nogood recording in MAC-CBJ SAT algorithms. Technical Report RT/04/2002, INESC, April 2002.

14. I. Lynce and J. P. Marques-Silva. An overview of backtrack search satisfiability algorithms. Annals of Mathematics and Artificial Intelligence, 37(3):307-326, March 2003. 
15. A. K. Mackworth. Consistency in networks of relations. Artificial Intelligence, 8(1):99-118, 1977.

16. J. P. Marques-Silva and K. A. Sakallah. GRASP: A new search algorithm for satisfiability. In Proceedings of the ACM/IEEE International Conference on ComputerAided Design, pages 220-227, November 1996.

17. M. Moskewicz, C. Madigan, Y. Zhao, L. Zhang, and S. Malik. Engineering an efficient SAT solver. In Proceedings of the Design Automation Conference, pages 530-535, June 2001.

18. P. Prosser. Domain filtering can degrade intelligent backjumping search. In Proceedings of the International Joint Conference on Artificial Intelligence, pages 262267, August 1993.

19. P. Prosser. Hybrid algorithms for the constraint satisfaction problems. Computational Intelligence, 9(3):268-299, August 1993.

20. P. Prosser. MAC-CBJ: maintaining arc consistency with conflict-directed backjumping. Technical Report 177, University of Strathclyde, Glasgow, Scotland, May 1995.

21. D. Sabin and E. Freuder. Contradicting conventional wisdom in constraint satisfaction. In Proceedings of the European Conference on Artificial Intelligence, pages 125-129, August 1994.

22. B. Selman and H. Kautz. Domain-independent extensions to GSAT: Solving large structured satisfiability problems. In Proceedings of the International Joint Conference on Artificial Intelligence, pages 290-295, August 1993.

23. R. M. Stallman and G. J. Sussman. Forward reasoning and dependency-directed backtracking in a system for computer-aided circuit analysis. Artificial Intelligence, 9:135-196, October 1977.

24. T. Walsh. SAT $v$ CSP. In Proceedings of the International Conference on Principles and Practice of Constraint Programming, pages 441-456, September 2000.

25. R. Zabih and D. A. McAllester. A rearrangement search strategy for determining propositional satisfiability. In Proceedings of the National Conference on Artificial Intelligence, pages 155-160, July 1988.

26. H. Zhang. SATO: An efficient propositional prover. In Proceedings of the International Conference on Automated Deduction, pages 272-275, July 1997. 